\title{
Clinical Laboratory Educators' Conference 2012 Abstracts
}

\section{SALT LAKE CITY, UT}

The following abstracts were presented during the 2012 American Society for Clinical Laboratory Science (ASCLS) Clinical Laboratory Educators' Conference February 23-25, 2012 in Salt Lake City, Utah. Abstracts are reviewed by appropriate representatives of the ASCLS Educational Scientific Assembly. They are the final authority in selecting or rejecting an abstract.

\section{Poster Presentations}

Clinical Laboratory Science Education: Developing a Regional Model

Linda Fell, MS, MLS (ASCP) ${ }^{\mathrm{CM}} \mathbf{S H}^{\mathrm{CM}}$; Marnie Imhoff, MBA, MLS(ASCP) ${ }^{\mathrm{CM}}$; Karen Honeycutt, MEd, MLS(ASCP) ${ }^{\mathrm{CM}} \mathrm{SM}^{\mathrm{CM}}$; Tiffany Colvin, BHS, MLS(ASCP) ${ }^{\mathrm{CM}}$, University of Nebraska Medical Center, Omaha, NE

To meet increased demand for certified clinical laboratory scientists, especially in rural areas and in regions that have experienced CLS program closures, the University of Nebraska Medical Center Clinical Laboratory Science Program has incorporated distance learning technologies into CLS education for 20 years. More than 320 students have received a baccalaureate degree in Clinical Laboratory Science/Medical Technology while completing the majority of their educational experience at a laboratory located at a distance from the metropolitan Omaha campus. This review of distance education in the CLS Program focuses on the progression of a model originally used in Nebraska and expanded to five partner universities in the Midwest. The Program uses a 3+1 format including an 11-week student laboratory phase completed at one of two academic sites followed by a nine-month clinical phase completed at one of 18 affiliate clinical sites. During these nine months, students complete a standardized curricula developed by the UNMC CLS Program Faculty via Internet-based technologies. Discussion includes use of various distance learning technologies and elements of successful academic and clinical site support. Benefits of a regional network include broad-based education with input from multiple instructors/sites for CLS students; the opportunity to work with students before hire and decreased costs and time for recruitment and orientation as well as access to resources for clinical sites; and an increase in well-prepared CLS graduates for the future for the profession with significant cost savings to universities in development of new programs.

Distance Education Course Design to Promote and Ensure Academic Integrity

Linda Graeter, PhD, MT (ASCP), Elizabeth Hertenstein, MS, MT(ASCP)SBB, Gideon Labiner, MS, MLS(ASCP) ${ }^{\mathrm{CM}}$, Elizabeth Warning, MS, MLS(ASCP) ${ }^{\mathrm{CM}}$, Susan Wilkinson, EdD, MT(ASCP), $\mathrm{SBB}^{\mathrm{CM}}$, ${ }^{*}$ Charity Accurso, PhD, MT(ASCP), University of Cincinnati, Cincinnati, $\mathrm{OH}$

Maintaining academic integrity in educational courses is a challenge faced in both traditional and distance learning settings. While many studies have assessed the prevalence of misconduct in either setting, the prevalence in one delivery method versus the other is contradictory. There are many best practices and course design elements that can promote and ensure academic integrity. In the University of Cincinnati Clinical Laboratory Science Distance Learning AS to BS degree completion program, we have incorporated best practices and experienced based elements into our curriculum in order to maintain academic integrity. Examples include honor codes, plagiarism and policies quizzes, discussion board formatting, test design, proctored examinations, and group design. We have also designed several protocols that assist us in reviewing student submissions and identifying acts of misconduct. These protocols will be discussed. While it is difficult to ascertain a drop in academic misconduct, the changes have made it more difficult to commit some violations and violations for plagiarism have decreased. The faculty are also more confident in their abilities to identify students at risk for such behaviors and in their 


\section{CLINICAL PRACTICE}

abilities to identify misconduct violations. Specific examples will be discussed.

\section{Evaluation of Critical Thinking Skills in Medical Laboratory Science Students \\ Nancy Bergeron MA, MLT, ART, University of Ontario Institute of Technology, Oshawa, Ontario}

The purpose of this study was to examine evidence of a change in critical thinking skills by fourth year in a University-based Medical Laboratory Science program. The importance stems from a lack of educational research in the Medical Laboratory Science field and the need for critical thinking skills. Critical thinking is an evolving process and many factors lead to this evolution. Age, educational background, high school courses, and teaching techniques all play a role. A crosssectional method of comparison of first and fourth year students was undertaken. The study incorporated a commercially available critical thinking instrument: the Health Sciences Reasoning Test. This test uses heath science related content, but is not discipline specific. A short survey, including age, gender, and education, selfacknowledgement of critical thinking skills, high school courses and educational strategies that develop these skills was included and used to compare scores. From a response rate of $56.4 \%$, the mean first and fourth year scores were 20.21 and 22.41 respectively (from a maximum score of 33). Using the score interpretation guide, the range of fourth year scores correlated with competence to strength in critical thinking ability. This research benefits students, the University program and the Medical Laboratory Science profession as a whole because the data confirmed that critical thinking skills improved to competence levels in the program curriculum. The research is still preliminary and should not be taken as representative of the program or profession due to its low participation numbers and evaluation of only one educational institution.

\section{Incorporating Genomics and Bioinformatics into the MLS Curriculum}

Daniel Golemboski, PhD, Bellarmine University, Louisville, KY

Traditionally, medical laboratory science students have been taught to identify bacteria using criteria such as morphology, immunological markers, and biochemical characteristics. Genome sequencing technologies have provided the information that enables a faster, more precise characterization of bacteria according to rRNA, DNA and other RNA sequences. Additionally, comparison of genomes from closely related bacteria makes it possible to determine why some strains are pathogenic, predict clinical outcomes of infections, and develop therapeutic strategies. In this exercise, students used computer databases and algorithms to analyze bacterial proteins and genes as an alternative to the traditional methods of identification and characterization. The students were presented with a case study involving a hospital outbreak of multidrug resistant Acinetobacter baumannii. The assignment focused on the ability to rapidly determine the antibiotic sensitivity pattern of the organisms. The students isolated DNA from the bacteria, designed primers for $16 \mathrm{~S}$ rRNA and the antibiotic resistance genes, carried out PCR reactions, and finally characterized the sequence of these amplified genes using a Basic Local Alignment Search Tool (BLAST) analysis. This exercise instructs students on a number of levels and provides the technical skills that are applicable to many molecular diagnostic protocols that are in use today. A similar exercise can be simulated by providing the gene sequences which are homologous to relevant genes in A. baumanii.

Infusing Diversity and Cultural Competence Principles in the Clinical Laboratory Curriculum Maria Torres Pillot, MA, MT(ASCP), Wichita State University, Wichita, KS

Health professionals need to understand and embrace diversity to demonstrate cultural competence. Cultural competence is a developmental process that encompasses multicultural awareness and appreciation for the value of diversity in the healthcare industry. The clinical laboratory educators have the opportunity to introduce the principles of cultural competence in the area of laboratory management. This project shows how the clinical laboratory curriculum of the medical technology program at Wichita State University was infused with a diversity and cultural competence module incorporated into the laboratory management course. This module introduces the broad definition of diversity and discusses the advantages of a diverse workforce in the clinical laboratory. A healthcare professional that demonstrates cultural competence may be able to communicate more effectively with patients and other healthcare professionals. Laboratory medicine and all healthcare professions have entered the 
globalization era and teams in labs around the world working on related tasks need not only to overcome language barriers but to understand cultural differences in order to increase the effectiveness of collaborative work. Accepting and embracing diversity is an important tool to become a successful laboratory professional. Each day we see an increasing culturally diverse population of students and laboratory workforce that could benefit from developing a set of cultural competence skills.

Leukemia Case Study Portfolios: An Effective Tool for Developing Critical Thinking Skills

Mary Jane Yue, MS, MT(ASCP), Cheryl Swinehart, MS, MT(ASCP), Christopher Desens, MLS(ASCP) ${ }^{\mathrm{CM}}$, University of Minnesota, Minneapolis, MN

When the University of Minnesota's Clinical Laboratory Sciences program expanded to a second campus and enrollment nearly doubled, it was not feasible to duplicate the way we were teaching leukemias in our Hematology Morphology course. Students had been individually examining slide sets of blood and bone marrow (both Wright and cytochemical stains) to correlate cell morphology with disease states. In the place of the slide sets, case study portfolios were created that could be used across performance sites. These portfolios included color prints made from digital images of the slides, case histories, a set of questions to use for discussion, and ancillary laboratory results (special hematology, cytogenetics, immunophenotype, and molecular diagnostics). Not all of the ancillary laboratory results had been included in the earlier case studies using the individual slide sets, so the portfolios provided a more complete and current view of the laboratory's role in diagnosing leukemias. Students were divided into groups and worked collaboratively to establish a diagnosis for each case. When they used case studies in the portfolios, we observed that they asked more questions of the faculty, and these questions demonstrated deeper critical thinking compared to the previous students who studied individual slide sets. The case study portfolios have shown to be an effective and low-cost method for teaching students the correlation between leukemias and laboratory results.

\section{Online vs. Hybrid Format for an Introductory CLS Course}

Donna J. Spannaus-Martin, PhD, MLS(ASCP) ${ }^{\mathrm{CM}}$, Janice Conway-Klaassen, PhD, MT (ASCP)SM, Cheryl
Swinehart, MS, MT(ASCP), Joanna George, MT(ASCP)SBB, University of Minnesota, Minneapolis, $\mathrm{MN}$

The Clinical Laboratory Sciences Program has taught an Introduction to Clinical Laboratory Science course for many years, using it as a recruitment tool for prospective students. As part of a Department of Labor grant, six online modules on laboratory testing (clinical microbiology, clinical chemistry, hematology, hemostasis, urinalysis, and transfusion medicine) were developed. For the first of these six modules, students were required to do both the online module and attend the face-to-face session. For the remaining five modules, students were given the option of either attending the face-to-face sessions or doing the online modules. Quizzes were given to students in both options. No difference was observed in quiz scores between delivery methods. Students provided feedback about the online modules vs. face-to-face sessions. Overwhelmingly, students preferred face-to-face sessions, particularly if hands-on activities were included. Although students appreciated the flexibility of online sessions in order to review disciplines at their own pace, they preferred being able to ask questions and get immediate responses from the faculty member that in-class sessions provide. Students also thought getting an opportunity to perform some laboratory testing gave them a much clearer idea of what it would be like to be a clinical laboratory scientist. The online modules will continue to be used, but face-to-face sessions will now be used for hands-on activities. The six online modules developed for this course are available at no cost to anyone interested in using them.

\section{Teaching Hematology Morphology Online...Can it Work?}

Mauri Brueggeman, MEd, MLS(ASCP) ${ }^{\mathrm{CM}}$, Mary Jane Yue, MS, MT(ASCP), Andrew R. Yue, MT(ASCP), Janice M. Conway-Klaassen, EdD, MT(ASCP), Cheryl Swinehart, MS, MT(ASCP), Stephen M. Wiesner, $\mathrm{PhD}$, MT(ASCP), University of Minnesota, Minneapolis, $\mathrm{MN}$

Hematology morphology is traditionally taught using a microscope and glass slides. Glass slides can break and fade over time and are a limited physical resource. To investigate the potential for statewide implementation of digital microscopy in laboratory science education, we tested its efficacy in our hematology morphology 
course. All slides from our library were digitally scanned and could be viewed from $2 \mathrm{X}$ to $830 \mathrm{X}$ using commercially available software. The digital slides could be examined by students in all manners similar to slides on a microscope with the exception of fine focusing. The majority of slides were accompanied by a case history and represented both normal samples and abnormal hematopathologies. All students completed the lecture portion of the course as one group. Students were randomly assigned to the treatment group of virtual microscopy (VM) for the laboratory portion and students not assigned to VM completed the laboratory portion using traditional microscopy (TM) 3 hours per week. Both TM and VM groups were balanced demographically. VM students accessed the digital slide library using a computer via the internet. Three qualitative surveys were conducted throughout the semester to assess perception, preparedness and expectations. Student scores on practical exams and final course totals were compared. While historical comparison demonstrates that overall grade distribution during the study did not differ from previous years, VM student performance was statistically better than TM student performance within the current cohort of students.

\section{The Use of Hospital Role Playing to Engage High School Students}

Lorraine Blagg, MLS(ASCP) ${ }^{\mathrm{CM}}$ SBB, Johns Hopkins Hospital, Baltimore, MD

The Johns Hopkins Hospital Pathology and Radiology departments partner with the Paul Lawrence Dunbar High School each summer for four weeks to provide high school students with the opportunity to see how allied health professionals contribute. A pathology session, "Trauma and the Blood Bank", used a presentation and role playing activity to engage learners and promote collaboration. The presentation included information on types of trauma with potential treatment options and reviewed the hospital employee's roles. The learners were divided into groups of four to five students each having a role: Trauma patient, Doctor, Nurse, Blood Bank Technologist, and Pharmacist. The trauma patient was given directions and a T-shirt to aid in acting out their trauma (motor vehicle accident, burn, and gunshot wound). The doctor and nurse worked together to determine the appropriate treatment and choose from options like taking X-rays, administering pain medication, performing surgery, drawing blood, and giving blood products. The Blood Bank Technologist was given a picture of $\mathrm{ABO} / \mathrm{Rh}$ typing and selected blood products accordingly. Each choice had consequences that were provided by the instructor. Props were created like blood bags (red blood cells, plasma, and platelets) and medications (candy) to enhance the sixty minute activity. The activity was received well by both the instructors and the learners. The students identified how healthcare is a team effort, how important communication is, and how each person has a role to play in patient care. At completion, the session was chosen to be modeled for other similar learning activities.

\section{Using an Online Instruction and Virtual Laboratories to Teach Hemostasis \\ Janice M. Conway-Klaassen, PhD, MT(ASCP)SM, Christopher Desens, MLS(ASCP) ${ }^{\mathrm{CM}}$, Phyllis Trcka, MT(ASCP), Cheryl Swinehart, MS, MT(ASCP), University of Minnesota, Minneapolis, MN}

Laboratory methodologies in Hemostasis have changed significantly over the past decades, from original testing methods of mixing reagents in tubes, incubation in a warm water bath and observing the tube for a clot to automated methodologies in multi-tasking instruments. Most medical laboratory instructors prefer to use manual or semi-automated methods to teach hemostasis so that students can "see" what is occurring during the testing method, but semi-automated instruments are no longer commercially available. Review of current workplace practices showed that basic hemostasis testing is now done with automated instrumentation, however the knowledge and principles of the assays are necessary to interpret testing and troubleshoot the instruments. We therefore needed to find a new way to teach Hemostasis. Working with an instructional design team versed in online education, we created five virtual Hemostasis laboratory exercises that mimic the manual methodologies along with web-based didactic instruction. Although we switched completely to a virtual teaching method in Hemostasis, we needed to evaluate the efficacy of this method. Several factors were evaluated including student performance on exams in lecture and laboratory courses; performance on specific test questions used for both methods; student satisfaction surveys; and clinical preceptor surveys. Preliminary data indicates that student outcomes for the virtual method group are equal to or better than the 


\section{CLINICAL PRACTICE}

historical method group and both student and preceptor feedback have been very positive on the value of the exercises.

Using Screen Recording and Audio to Support Student Learning in a Hybrid Hematology Course Sandra M. Weiss, EdD, MLS (ASCP) ${ }^{\mathrm{CM}}$, Neumann University, Aston, PA

Technological improvements, such as capturing quality screen recordings along with audio and webcam video, permit an easy method for taping lectures, prelaboratory instructions, and teaching cell identification. Today, students expect to learn at convenient times using multimedia communication systems and accessing diverse delivery methods. This poster presentation session will concentrate on the development of screencasting lectures, pre-laboratory instructions, cell identification and morphology instruction using Camtasia Studio 7.1. This software permits easy recording of your PowerPoint, Desktop, animations, and videos as if the instructor is presenting live. Advantage to this type of recording is that it can be produced and shared in any format, such as iPhone, iPod, YouTube, and iPad. Students have the ability to pause the recording to take notes and have the ability to review the recording at a later time using a device of their choosing. This method supports lecture and laboratory, which permits the student time; particularly, in laboratory to practice, as well as, the ability to enhance student-teacher interaction. Assessing learning in higher education includes various course-specific indicators, such as instructor-designed tests, tasks, projects, as well as, student and instructor perceptions. Students in the hybrid hematology course were evaluated on their competency in blood cell identification, hemostasis and body fluids using various methods, such as the CellaVision DM software, electronic portfolio, and unknowns. Students' learning perceptions were assessed using an online survey. Students' scores on practical exams were compared with scores from previous years. Students in the hybrid course scored higher on all practical exams.

\section{Technology Demonstrations}

The Design of an Online Program Orientation Course

Charity Accurso, PhD, MT(ASCP), Linda Graeter, $\mathrm{PhD}, \mathrm{MT}(\mathrm{ASCP})$, Elizabeth Hertenstein, MS, MT(ASCP), SBB, Elizabeth Warning, MS,
MLS(ASCP) ${ }^{\mathrm{CM}}$, Susan Wilkinson, EdD, MT(ASCP), $\mathrm{SBB}^{\mathrm{CM}}$, Gideon Labiner, MS, MLS(ASCP) ${ }^{\mathrm{CM}}$, University of Cincinnati, Cincinnati, $\mathrm{OH}$

Promoting student success in a distance education environment requires strategies that are specifically designed for online delivery. Student satisfaction and success is established in the first few weeks of class, therefore it is important to address concerns about their new environment early on. Incoming students must be well prepared technologically, including pre-acclimation to the online course model that will be utilized. In our program, new students must successfully complete an Orientation Course (ORT) just prior to their first term in the program. The ORT is delivered using Blackboard; its structure parallels that of the program's courses. Students complete a series of interactive modules that provide an overview of the program; establish the appropriate settings on the student's home computer; teach the computer and Blackboard skills that will be required; familiarize the student with our course management system; establish a foundation for writing skills and test the student's abilities using a series of assessments. Students who might need training beyond completion of the ORT are identified and provided with additional support. During the Technology Demonstration, the ORT will be reviewed from both a student and instructor perspective. The various learning activities that are used to establish competencies will be shown along with the use of advanced Blackboard tools such as moderated discussion settings and linking instructional tutorials. The evolution process resulting in our current ORT will be summarized, including lessons learned.

\section{Teaching the Exclusion Method of Antibody Identification with an Interactive Computer Program \\ Joanna George, MT(ASCP)SBB, Jason Hill, MS, University of Minnesota, Minneapolis, MN}

In a Blood Bank curriculum, teaching antibody identification with the red cell panel exclusion (crossout) method is a time consuming exercise, often requiring one-on-one instruction with the student. At the University of Minnesota, program expansion at both the Minneapolis and Rochester sites necessitated a new method to deliver this content. An interactive computer based program has been developed whereby students can work through a red cell panel phenotype 
sheet displaying patient results. After some initial instruction and orientation, students will sequentially move through each cell of the panel, crossing off individual antigens with a mouse click. Immediate feedback is given for each correct and incorrect move, and progressing out of order or skipping ahead is not allowed. Once all the crossing out is complete, the student is allowed to identify the most probable antibody present with feedback given. Additional panels will be presented sequentially, designed to demonstrate a variety of situations and rules related to antibody identification. This program will be implemented in spring 2012 as a requirement before performing the first wet panel in lab. A comparison of student scores on standard assessment questions will be made with previous classes. A student satisfaction and feedback survey will be conducted and an instructor survey will assess student readiness and reduction in one-on-one tutoring. A proposal to present findings will be made at a future meeting.

\section{Use of Stereographic 3D Video for Enhancing the Learning Experience of Phlebotomists in Training Kristin Landis-Piwowar, PhD, MT(ASCP), Aaron J.} Bird, PhD, Patricia Wren, PhD, MPH, Florence Dallo, $\mathrm{PhD}, \mathrm{MPH}$, Oakland University, Rochester, MI

Laboratory educators continually strive to adopt effective teaching approaches to yield improved student learning outcomes. While there is no "holy grail" teaching approach that can be universally applied, new technologies combined with traditional classroom-based learning can bring about increases in knowledge, skill, attitude, and perception. One such technology is stereographic 3D. Recent research conducted at Oakland University has shown that information delivered via stereographic 3D video improves learners' ability to remember information and changes viewers' perceptions. Because phlebotomy is critical to the medical laboratory scientist with regard to patient identification and the acquisition of a high-quality specimen, we developed new 3D video content detailing the steps of a safe and routine blood draw. Learners experience the $3 \mathrm{D}$ video in a semi-sensory deprivation system that obstructs surrounding sights and sounds thereby increasing viewer's attention to the $3 \mathrm{D}$ video and audio content. While use of video to teach blood draw is not novel, presenting this information through immersive $3 \mathrm{D}$ video is. The video includes graphics depicting physiological events at the puncture site including vein compression following tourniquet application, blood flowing into a needle, formation of a platelet plug and fibrin clot, formation of a hematoma following improper technique, etc. This stereographic $3 \mathrm{D}$ experience informs students learning phlebotomy of the best possible practices and importantly why those practices are critical to both patient and specimen.

\section{Using a Web-Based Module for Preceptor Training} Patricia J. Brennecke, MT(ASCP), Janice M. Conway-Klaassen, PhD, MT(ASCP)SM, Donna Spannaus-Martin, PhD, MLS(ASCP) ${ }^{\mathrm{CM}}$, University of Minnesota, Minneapolis, MN

Over the past three years with the support of a Department of Labor grant, Minnesota has dramatically increased enrollments in its laboratory education programs. At the University of Minnesota we needed to find ways to facilitate the clinical experience for the both students and clinical sites. With support from the grant we developed a set of web based training modules to help clinical preceptors work with students in a more efficient manner which would hopefully allow them to take more students for training. Preceptor training modules described the educational background of our students and provided an understanding of a clinical experience including lessons on 1) Roles and Definitions; 2) What is Entry Level; 3) Student Affective Domain; 4) Positive Environments for Learning; and 5) Student Evaluations. We first held a number of synchronous sessions for our clinical site personnel again beginning with a discipline specific overview of didactic content and student laboratory activities along with a discussion of expectations for clinicals. The final sessions focused on preceptors behaviors and evaluations processes during the clinical experience. These discussions where then incorporated into the final modules. Because the focus is on students applying what they have learned to the real world and not on theory training at the bench the sites, we have been able to move to this new model of a clinical experience while maintaining our program outcome metrics. 